\title{
Les interrogations du système fédéral allemand
}

Jürgen Baumert, Olaf Köller, Dirk Konietzka et Kai Schnabel

Traducteur : Dominique Gélin

\section{OpenEdition}

\section{Journals}

Édition électronique

URL : http://journals.openedition.org/ries/3160

DOI : $10.4000 /$ ries. 3160

ISSN : 2261-4265

Éditeur

Centre international d'études pédagogiques

Édition imprimée

Date de publication : 1 septembre 1997

Pagination : $27-45$

ISSN : 1254-4590

\section{Référence électronique}

Jürgen Baumert, Olaf Köller, Dirk Konietzka et Kai Schnabel, « Les interrogations du système fédéral allemand », Revue internationale d'éducation de Sèvres [En ligne], 15| 1997, mis en ligne le 04 juillet 2013, consulté le 20 avril 2019. URL : http://journals.openedition.org/ries/3160 ; DOI : 10.4000/ ries.3160

Ce document a été généré automatiquement le 20 avril 2019

(c) Tous droits réservés 


\title{
Les interrogations du système fédéral allemand
}

\author{
Jürgen Baumert, Olaf Köller, Dirk Konietzka et Kai Schnabel \\ Traduction : Dominique Gélin
}

1 Il n'est pas possible de comprendre les discussions portant sur les structures et les transformations du système éducatif allemand, de comprendre comment sont prises les décisions politiques et comment les choses se réalisent dans la pratique en République fédérale, depuis sa fondation en 1949, sans prendre en compte précisément la nature fédérale de la République ni se référer à la «souveraineté des Länder» qui la composent en matière d'éducation (art. 30, art. 83 et suivants de la Loi fondamentale). Les réformes importantes du système scolaire en République fédérale - l'enseignement public étant prépondérant - ne peuvent être mises sur pied qu'avec le consensus des responsables gouvernementaux des seize Länder. La Conférence permanente des ministres de l'Éducation qui était à l'origine, en 1949, une simple réunion de travail, sur la base du volontariat, est rapidement devenue, pour les questions de politique éducative, un instrument de coordination au-delà des frontières régionales. On y délibère, le plus souvent en comités, sur les thèmes concernant la politique éducative et on y élabore des recommandations qui devront être adoptées à l'unanimité en séance plénière. Elles prennent de ce fait un caractère obligatoire pour les gouvernements des Länder sans être pour autant des lois au sens propre du terme. Cette nécessité d'aboutir à un consensus conduit obligatoirement à prendre des résolutions qui laissent aux gouvernements des Länder une liberté d'action dans leur politique, si bien que des différences considérables existent entre les Länder, dans ce domaine, et que, par voie de conséquence, les controverses sur la politique à suivre en matière d'éducation ne sont pas aussi accentuées d'un Land à l'autre.

2 Ceci rend difficile le choix des thèmes qui peuvent être considérés comme ayant une importance particulière dans les débats sur la politique et les sciences de l'éducation. C'est la raison pour laquelle nous nous sommes limités, pragmatiquement, aux thèmes particulièrement récurrents à long terme quand on suit l'évolution des phénomènes 
socioculturels et qui concernent, pour cette raison, le système scolaire de toute la République fédérale. Doivent être abordées en outre les questions qui ont également une grande importance pour le processus d'intégration dans la Communauté européenne. C'est, à notre avis, le cas pour les thèmes suivants :

- l'avenir de la formation professionnelle (la « crise du système dual »);

- la réforme du système scolaire dans les nouveaux Länder;

- le renforcement de l' " autonomie des écoles ";

- le contrôle élargi de la réussite dans le système scolaire.

\section{Crise du système dual ?²}

3 En Europe de l'ouest et aux États-Unis, la qualification professionnelle des jeunes et leur intégration dans le marché du travail sont devenues, ces dernières années, des questions importantes, étant donné le taux élevé de chômage touchant les jeunes, sur lesquelles s'est fixée tout particulièrement l'attention publique. Dans cette perspective, les regards se sont portés, avec toujours plus d'insistance, sur les conditions institutionnelles dont dépend la relation entre la formation professionnelle et une intégration réussie et stable des jeunes dans le marché du travail. On accorde fréquemment, dans les études comparatives internationales, une attention particulière au système allemand de formation professionnelle car il semble être particulièrement apte non seulement à donner une qualification professionnelle à une grande partie d'une classe d'âge mais également de l'intégrer, à la suite de cette formation, sans hiatus et de façon stable au marché du travail. Mais en même temps que le système dual devenait un "produit phare d'exportation " potentiel, une vive discussion se développait en Allemagne, portant sur la situation présente et sur les perspectives d'avenir de la formation professionnelle ${ }^{3}$. Au centre de ces débats: l'idée que, dans le domaine de la formation scolaire et professionnelle, le comportement des nouvelles classes d'âge répond à un modèle différent. Il convient d'abord de tracer une brève esquisse des caractéristiques qui sont au cœur du «système dual» de formation, puis d'aborder les perspectives récentes d'évolution de la formation professionnelle et, pour finir, la question d'une éventuelle tentative de réforme.

\section{Le système dual}

4 Même si l'on considère que les fondements de la formation professionnelle, effectuée essentiellement en entreprise et associée à un enseignement dispensé dans un établissement public, ont été mis en place à la fin du XIX siècle, le concept de «système dual » est apparu avec une expertise de la Commission allemande pour le système scolaire et éducatif (Deutscher Ausschuss fur das Erziehungs- und Bildungswesen) concernant le système scolaire et éducatif professionnel de la République fédérale, en 1964. L'organisation de l'apprentissage en Allemagne est définie dans cette expertise par la dualité des lieux d'apprentissage (entreprise où a lieu la formation et école professionnelle). Cependant, le critère du lieu d'apprentissage à lui seul est insuffisant pour définir le système dual. Ce qui est déterminant pour ce système dans sa forme actuelle est la loi sur la formation professionnelle, datant de 1969, constituant la base 
juridique, en vigueur jusqu'à aujourd'hui, qui régule et dirige la formation professionnelle duale. Selon cette loi, le système dual repose sur trois principes fondamentaux :

- la liberté de contrat lors de l'établissement de relations de formation;

- la compétence de l'État en ce qui concerne le règlement de la formation professionnelle ;

- les structures corporatives pour ce qui est des processus de planification, de décision et de contrôle essentiels4.

5 L'organisation et la réglementation de la formation professionnelle dans le système dual sont, de cette manière, solidement intégrées dans l'ensemble des relations industrielles. Les règlements concernant la formation sont ainsi négociés, pour l'essentiel, entre les instances patronales, les syndicats représentant les travailleurs et les institutions publiques (avant tout l'Institut fédéral pour la formation professionnelle - Bundesinstitut für Berufsbildung) $)^{5}$. La formation professionnelle duale est aussi, de la sorte, largement normalisée et standardisée au niveau fédéral. Il est également significatif, dans la logique de cette centralisation, que les examens de fin de formation soient organisés au niveau central par les différentes chambres compétentes ce qui permet d'obtenir un certificat de fin de formation reconnu partout. Cet aspect est aussi important pour la mobilité professionnelle que la macrostructure du marché du travail ${ }^{6}$. Le système dual de la formation professionnelle n'est par conséquent en aucun cas défini par le seul critère des lieux d'apprentissage où elle est pratiquée. Si l'on veut tenter d'exporter ce système dual dans d'autres pays, il faut respecter aussi, d'une manière appropriée, les principes spécifiques de ce mode de formation professionnelle, observer la même politique de réglementation - au moins lorsqu'on espère par là introduire en même temps un cadre institutionnel et créer des conditions telles que de jeunes adultes puissent s'intégrer avec succès dans la formation professionnelle et dans le marché du travail.

L'évolution des systèmes de l'emploi et de la formation dans les années quatre-vingt et quatre-vingt-dix a également considérablement modifié les conditions, le cadre dans lequel fonctionne le système dual. D'un côté, des décalages par rapport aux lieux d'apprentissage, une nouvelle image, une façon nouvelle d'envisager le travail et de nouvelles exigences en matière de qualification, de l'autre, de nouveaux modes de comportement, en ce qui concerne la formation scolaire et professionnelle, sont ainsi des facteurs importants qui placent le système dual devant de nouveaux défis, présents et à venir, s'ils ne le menacent pas sérieusement à long terme dans sa substance même.

\section{Une nouvelle conception de la qualification}

7 Pour de nombreuses activités professionnelles, les exigences concernant le niveau de qualification se sont élevées, conditionnées par les changements de structures des métiers et l'importance grandissante de la technologie dans les profils professionnels ; on fait de plus en plus appel à des compétences théoriques et analytiques ${ }^{7}$. Les contenus de l'enseignement professionnel ont eu tendance ces dernières années, de façon analogue, à prendre un caractère plus analytique et, de ce point de vue donc, moins spécifique à tel ou tel métier particulier. Depuis les années quatre-vingt, le système dual a réagi à ces changements dans le système de l'emploi : les images des différents métiers faisant l'objet d'une formation professionnelle ont été successivement modernisées. La tendance à modifier le concept de qualification dans la formation professionnelle est également remarquable. Ainsi, dans la nouvelle classification des métiers de la métallurgie et de l'électricité de 1987, le concept de «métier » a été au moins en partie remplacé par la 
définition de principaux «secteurs professionnels» avec différentes orientations spécifiques à chaque spécialité. La formation doit alors permettre d'acquérir des qualifications-clés plus abstraites, son objectif est une "capacité d'action" professionnelle individuelle qui permette de planifier une tâche, de l'exécuter et de la contrôler de façon autonome. On retrouve des objectifs formulés de manière identique dans les principes fondamentaux de la nouvelle nomenclature des métiers de la bureautique, où l'on va encore plus loin : dans l'enseignement professionnel de ce secteur l'acquisition de compétences personnelles et sociales occupe une place centrale.

8 Avec la modification et, dans certains cas, l'élévation du niveau d'exigence de la qualification - en particulier, avec l'importance croissante prise par les qualifications clés à la place des capacités spécifiques à une spécialité se pose à nouveau la question de savoir quelles institutions seraient les plus à même d'apporter ces qualifications. Si autrefois les écoles professionnelles avaient clairement reçu pour mission de transmettre les bases d'un savoir théorique - tandis que l'entreprise avait pour tâche l'insertion dans la pratique professionnelle -, ces dernières années ce qu'on appelle les lieux de formation du troisième type, sous la forme d'ateliers de formation pour les grandes entreprises et de lieux de formation communs à plusieurs entreprises dans le secteur de la formation artisanale, ont pris en charge des objectifs importants de qualification à l'intérieur du système dual. Si cependant les firmes subdivisent de plus en plus certains domaines de la formation pratique en entreprise en secteurs de formation autonomes, la partie de la formation qui se déroule dans l'entreprise prend, de plus en plus, un caractère systématique, analytique, et il est de moins en moins possible de considérer qu'il s'agit d'une simple formation pratique. Une part croissante de bases théoriques de la formation est enseignée également de la sorte à l'extérieur de l'école professionnelle. C'est la raison pour laquelle, ces dernières années, à la suite de ces décalages des structures institutionnelles, la fonction et la légitimité des écoles professionnelles ont été soumises à une forte pression et mises en cause en tant que structures d'accompagnement théorique de la formation pratique en entreprise.

Mais la formation professionnelle dans le système dual se trouve encore plus fortement confrontée à de nouveaux défis du fait du changement de stratégie de formation des jeunes. Dans les années cinquante et soixante, le système de formation dual était relativement homogène, qu'il s'agisse des conditions d'entrée (selon l'origine sociale et la formation scolaire des apprentis) ou des carrières embrassées à la fin de la formation. La signification sociale du système dual tenait au fait, en particulier, qu'il rendait accessible à des élèves issus de la Volksschule, d'un niveau au-dessus de la moyenne, l'accès à un emploi qualifié. Aux élèves issus d'un cursus plus long ou d'un niveau plus faible, on offrait à la fin de l'apprentissage des carrières différentes, clairement séparées. Cependant dans les années quatre-vingt, au plus tard, des modes de comportement nouveaux ont fait leur apparition dans la formation professionnelle et, par là, le rapport entre l'enseignement général et l'enseignement professionnel a subi des modifications, de façon durable. Ainsi, en raison de la diminution du nombre des élèves issus de la Volksschule puis de la Hauptschule (passé de plus de $70 \%$ à environ un tiers des effectifs en l'espace de trente ans), le lien traditionnellement étroit entre ces écoles et l'apprentissage a perdu de son importance. Par ailleurs, des diplômés de l'Abitur de plus en plus nombreux - leur nombre a plus que doublé dans le même laps de temps - se pressent sur le marché de la formation duale et réduisent, de ce fait, les chances des autres élèves d'accéder, au sortir de l'école, à des métiers qui exigent une meilleure qualification et 
offrent de meilleurs perspectives d'avenir. Le système dual pourrait obtenir le plus grand succès grâce à l'intégration de différentes couches de la population scolaire, comme le démontrent aussi bien les chiffres absolus que les quotas de la formation professionnelle ${ }^{8}$. Mais c'est justement la combinaison de l'Abitur et de l'apprentissage qui confronte le système dual à un profond problème, car le recrutement des apprentis n'est qu'un aspect du système dual ; l'autre aspect concerne les débouchés, l'entrée dans une carrière et la continuité de l'emploi à la fin d'une formation professionnelle. Ce sont précisément les apprentis détenteurs d'un plus haut niveau de formation générale (Abitur) qui considèrent souvent, de prime abord, l'apprentissage comme un stade transitoire sur le chemin des études supérieures. Ainsi, $29 \%$ des étudiants qui ont commencé leurs études en 1990 avaient déjà suivi un apprentissage auparavant. Et presque $50 \%$ des diplômés ayant suivi un apprentissage après l'Abitur poursuivent des études ${ }^{9}$. Au total, les modes de formation et les emplois occupés par les dernières générations se caractérisent par :

- l'allongement de la période d'apprentissage et de la phase de transition école-métier ;

- le report du moment où l'on entre dans le système de l'emploi à une période plus tardive ;

- la multiplicité des épisodes de formation scolaire et professionnelle avec un retour à des phases de formation supplémentaire après une phase d'activité professionnelle.

La conséquence directe de cette évolution est une hétérogénisation des carrières professionnelles avec une discontinuité plus forte de l'emploi ; le phénomène selon lequel des ouvriers spécialisés abandonnent les métiers qu'ils ont appris s'accentue.

11 À côté de cette demande croissante de formation professionnelle de la part d'élèves ayant suivi des cursus qui les menaient plus loin, cette hétérogénisation sociale concernant les personnes qui entrent dans le système de la formation professionnelle s'est également amplifiée en raison de l'intégration des femmes et, ce qui est loin d'être négligeable, de l'intégration des jeunes étrangers. Les distinctions faites au sein du public en fonction de la formation préparatoire, du sexe, de la nationalité et de l'âge ont cependant contribué à accentuer les phénomènes de sélection et de refoulement interne et, ce faisant, les formes de segmentarisation et de rejet dans le système dual. Les entreprises qui participent à la formation réagissent ainsi de plus en plus à l'hétérogénisation des candidats à l'apprentissage en ayant recours à des processus de sélection spécifiques à chaque spécialité dans le choix des apprentis. De ce fait, les résultats scolaires de l'enseignement général sont des critères qui pèsent de plus en plus lourd pour accéder au système dual, ce qui a tendance à désavantager à nouveau les élèves issus de la Hauptschule - clientèle traditionnelle de ce système. De cette manière se mettent aussi en place des mécanismes d'orientation plus ou moins persistants, que l'on voit apparaitre «sous la forme de segments du marché de la formation professionnelle concernant un ou, dans certains cas, plusieurs métiers isolés, réservés à des élèves qui ont obtenu tel niveau à l'issue de leur scolarité et d'un sexe différent ${ }^{10}$ ». Il faut considérer dans cet esprit la tendance de plus en plus marquée à la concurrence interne, qui aboutit à un refoulement des candidats, comme un problème structurel dans le système dual. Les conséquences de cette segmentation du marché de la formation professionnelle ne se limitent pas au fait qu'on trace une frontière qui barre la route à une partie des candidats, on voit également des déséquilibres structurels qui se manifestent par exemple par le fait que, malgré le grand nombre des "candidats qui n'ont pas encore bénéficié d'un apprentissage», de nombreuses places de travail restent, pour autant, inoccupées.

12 Les bases socio structurelles et socioculturelles du système dual semblent, elles aussi, être menacées à la longue. L'attrait de la formation professionnelle dans le système dual était 
étroitement lié dans le passé à la structure hautement sélective, socialement, de l'enseignement général, comme l'a fait remarquer Lut ${ }^{11}$. Tant que des barrières institutionnelles restrictives ont été opposées aux ambitions professionnelles d'une grande partie d'une classe d'âge du fait des capacités très limitées du système d'enseignement général, le chemin de l'ascension professionnelle, pour les couches sociales n'appartenant pas à la bourgeoisie, passait par la formation professionnelle dans le système dual. La structure de classe spécifique du système scolaire considéré dans son ensemble était ainsi la condition essentielle qui conférait au système dual de formation son rôle capital dans le domaine social. Le développement et l'ouverture sociale de l'enseignement général, ces dernières décennies, a vidé de son contenu la voie traditionnelle qui permettait, à partir de la Volksschule et de la Hauptschule, de passer par la formation professionnelle pour accéder à une carrière de " moyenne envergure » et, de ce fait, a brisé un facteur essentiel du système dual : la fonction qui consiste à apporter un statut professionnel, laquelle a eu tendance à se décaler de l'ascension au sein de l'entreprise vers les examens de fin de cycle de l'enseignement général ${ }^{12}$. La dernière conséquence étant que le système de la formation professionnelle n'attire plus que ceux qui ont échoué dans le système d'enseignement général. Les élèves qui quittent l'école avec un niveau faible ou moyen ont tendance à représenter un groupe sélectionné de façon négative parmi la population. C'est à peine s'ils constituent encore ce groupe «performant » dans lequel les entreprises recrutaient auparavant par l'avancement, avec la pratique du métier, le gros du personnel de techniciens exerçant la responsabilité de contremaître. Les conséquences à moyen terme de cette "tendance à la raréfaction croissante, quantitative et qualitative, de la main d'œuvre parmi les techniciens engendrée par la logique méritocratique ${ }^{13}$ » constitue finalement aussi un changement de structure, profond, des systèmes de l'emploi et des structures organisationnelles dans l'économie et les entreprises. La possibilité de progresser à l'intérieur de l'entreprise à la suite d'une formation professionnelle dans le système dual est, peu à peu, démantelée en raison du manque de personnel compétent.

\section{Stratégies de réforme}

13 On considère fréquemment que, pour faire baisser la pression croissante que représente la préférence accordée aux élèves issus de l'enseignement général pour l'accès à la formation professionnelle, le développement de la flexibilité des parcours de formation serait une stratégie sensée de la politique de l'éducation. L'attrait qu'exerce socialement une formation professionnelle dépend en effet essentiellement, dit-on, des passerelles menant aux niveaux supérieurs du système scolaire. Les projets de réforme des systèmes de formation s'orientent, dans cet esprit, vers un élargissement des droits formels des apprentis ayant achevé leur formation professionnelle, en particulier l'ouverture de l'accès aux formations professionnelles du supérieur pour les élèves issus du système dual. A en croire certains, des parcours permettant après une formation professionnelle de poursuivre une formation dans d'autres systèmes, une amélioration de la perméabilité entre les différentes formations et, dans certains cas, des passages possibles entre des secteurs de la formation professionnelle et d'autres secteurs de l'enseignement général, seraient, avant tout, des mesures appropriées ${ }^{14}$. parviennent pas à sortir d'une constellation paradoxale : les incitations qui permettent, 
dans un premier temps, de susciter un plus grand afflux de candidats vers la formation professionnelle ont tendance, quelque temps après, à entraîner à nouveau un abandon du métier appris. Elles manquent leurs objectifs, ne réglant pas les problèmes fondamentaux que représentent, dans le cadre de l'expansion du système scolaire ces trente dernières années, le changement de structure du travail et de comportement vis-à-vis des formations et les mécanismes d'attribution d'un statut. La dynamique des changements de comportement dans le domaine de la formation renvoie aux mécanismes centraux des chances laissées aux individus dans le domaine social et professionnel qui, de plus en plus, lie d'une façon rigoureuse l'accès à des places plus élevées dans le système de l'emploi, des places qui suscitent des ambitions, à la détention de certifications - avant tout scolaires - d'un niveau plus élevé. C'est pourquoi la formation professionnelle dans le système dual n'aura vraiment d'avenir que s'il est mis un terme à cette tendance. Ceci est d'autant plus vrai que la réussite du système dual dans le passé était étroitement liée à la perspective d'une progression dans le domaine professionnel ${ }^{15}$. Les incitations à suivre la voie d'une formation professionnelle dans le système dual ne peuvent donc se stabiliser que si on offre aussi des possibilités, des chances de progression dans la profession à la suite de la formation. Mais cela signifie que l'organisation du travail au sein de l'entreprise, les structures concernant les salaires et les carrières, les modèles de l'organisation du travail et de l'évolution du personnel doivent également être réformés, afin qu'à nouveau les élèves qui quittent le système scolaire se sentent davantage attirés par une formation professionnelle dans le système dual. Pour que le travail de technicien soit mieux accepté, il faut par conséquent que des changements aient lieu dans la façon de concevoir la production et le travail. Cela suppose, concrètement, de "valoriser clairement le travail productif et de transférer bien davantage, sur les lieux de travail, responsabilités et pouvoirs de décision ${ }^{16}$ ». Des réformes isolées se limitant aux chemins d'accès et aux droits de la formation professionnelle dans le système dual ne suffiraient guère, par contre, à rendre à plus long terme la formation professionnelle plus attirante socialement en Allemagne et encore moins d'en améliorer la qualité.

\section{Reconstruction du système scolaire dans les nouveaux Länder $^{17}$}

Avec la chute du Mur et la réunification, il était nécessaire dans les nouveaux Länder de prendre une décision pour jeter les bases de la structure du futur système scolaire de l'exRDA. Quand on regarde en arrière il est dès à présent frappant de voir que le système scolaire différencié des anciens Länder de l'Ouest a été repris sans grandes résistances au début de l'année 1991/1992 $2^{18}$. Au cours de ce processus de transformation structurelle, trente années d'évolution dans les anciens Länder ont été rattrapées en un temps très court. Le nombre de lycéens dans les nouveaux Länder correspond entre temps à peu près à celui des anciens Länder. Seule la Hauptschule peut être considérée comme la "grande perdante » de ce processus, le nombre relatif d'élèves qui la fréquentent étant nettement en dessous de celui des anciens Länder.

Le système éducatif institutionnalisé de l'ex-RDA avait pour idée directrice l'épanouissement de ce qu'il était convenu d'appeler la personnalité socialiste qui devait se développer dans tous les domaines avec une formation de base s'appuyant sur un large éventail de matières scientifiques. Cette maxime, ce principe éducatif s'intégrait dans le cadre collectiviste d'un système scolaire unitaire étendu sur dix ans, dans lequel toute 
disparité était réduite au minimum et où la formation de base d'une promotion entière devait atteindre un niveau assez élevé pour suivre un cycle long.

L'étude des promotions effectuée, sur le modèle d'une coupe longitudinale, par l'Institut Max-Planck pour la recherche sur l'éducation, en collaboration avec l'Institut pour la pédagogie des sciences exactes à Kiel, étude unique en son genre intitulée "Cursus et développement psycho-social des adolescents" fournit une documentation sur le passage, dans les nouveaux Länder, de ce système unitaire au système différencié. La recherche a commencé en 1991, immédiatement après le changement de système, si bien que les premières données sont encore une conséquence du système scolaire unitaire. D'autres données ont été recueillies en 1992 et 1995 à la fin de premier cycle du secondaire. Au total plus de 9000 élèves, filles et garçons, y ont participé dans les anciens et les nouveaux Länder. À chaque collecte de données, les résultats obtenus dans différentes disciplines (biologie, mathématiques, physique) ont été mesurés à l'aide de tests de réussite spécialement validés pour les curricula. Ces tests permettent d'analyser les conséquences du processus de transformation en prenant comme repère «la » variable centrale concernant les élèves, c'est-à-dire les résultats scolaires. Il est indiscutable, avant comme après, que l'efficacité de l'école - au niveau national ou international - est mesurée dans une première démarche à partir du niveau de qualification des élèves ${ }^{19}$.

18 Les hypothèses sur lesquelles étaient centrées les analyses des premières données concernant les résultats obtenus par les élèves faisaient encore référence, de façon explicite, aux différences entre le système scolaire unitaire de la RDA et le système différencié de l'ancienne République fédérale allemande ${ }^{20}$.

- étant donné que l'enseignement des mathématique et des sciences exactes était particulièrement privilégié dans l'ex-RDA, les élèves des nouveaux Länder devaient obtenir des résultats plus élevés ;

- en raison du caractère unitaire de la POS (Polytechnische Oberschule), dans lequel tous les élèves d'une promotion quels que soient leurs résultats étaient réunis dans la même classe, pour y recevoir le même enseignement et en tenant compte du fait que le soutien apporté aux élèves ayant les moins bons résultats était un objectif important ${ }^{21}$ : l'hétérogénéité des résultats devait, au total, être moins grande dans les Länder de l'ex-RDA; les élèves des nouveaux Länder qui avaient des résultat médiocres devaient se trouver plus nettement avantagés, par rapport aux jeunes des anciens Länder, que ceux obtenant les résultats les plus élevés.

19 Les analyses correspondantes confirment largement ce qu'on attendait, les élèves des nouveaux Länder obtenant, de manière significative, des résultats plus élevés dans les disciplines prises en compte, les écarts étant plus importants en biologie et en physique, et plutôt moins importants en mathématiques. Ces différences sont dues, en premier lieu, aux élèves obtenant des résultats médiocres qui se montrent nettement avantagés par rapport à ceux des anciens Länder. Pour les élèves les plus performants, on ne constate pas de différence significative entre les anciens et les nouveaux Länder. De même, conformément à ce qui était attendu, on peut constater une plus grande hétérogénéité des résultats scolaires dans les anciens Länder.

Ces éléments de l'enquête sont bien le reflet des conséquences que peuvent avoir différents systèmes scolaires et différents types de société sur les résultats scolaires en mathématiques et dans les disciplines scientifiques. Les analyses complémentaires de ces données servaient avant tout à apporter une documentation sur les conséquences que le 
processus de transformation avait eu sur les résultats scolaires des élèves des nouveaux Länder. Ces analyses longitudinales montrent, cum grano salis, que les résultats scolaires se sont largement équilibrés dans les anciens et dans les nouveaux Länder, ce qui correspond également aux autres enquêtes, menées à grande échelle, sur les résultats scolaires ${ }^{22}$. Ces observations sont-elles des indicateurs montrant que le système différencié introduit récemment a une efficacité moindre ou bien font-elles apparaître des changements dans des variables comme la motivation des élèves et, d'une façon générale, leur attitude vis-àvis de l'école ? Jusqu'ici, la question reste ouverte.

Dans les nouveaux Länder la transformation du système scolaire et le passage, globalement, à un autre type de société a sans conteste également eu une influence sur le psychisme des adolescents. Pour apporter la documentation nécessaire à ce sujet, des questionnaires ont été également mis en circulation, dans le cadre de cette enquête, concernant les réactions émotionnelles des élèves (crainte de l'échec, désarroi, peur de l'avenir etc.). Les données collectées montrent clairement à quel point ont pu peser, émotionnellement, sur les jeunes de l'ex-RDA au début de l'enquête (1991), une plus grande crainte de l'échec et une plus grande peur de l'avenir qui s'étendaient seulement, de façon très nette, sur une période d'un an. Malgré toutes les incertitudes, il semblerait qu'il y ait eu ici une " adaptation émotionnelle » très rapide aux nouvelles conditions de vie.

\section{Autonomie des écoles ${ }^{23}$}

Comme dans d'autres États européens, la revendication d'un plus grand transfert de responsabilités en faveur de l'école, dans la République fédérale, n'est en aucun cas un phénomène nouveau. En 1954 déjà, Hellmut Becker, dans un article abondamment cité24, avait réclamé une plus grande liberté pour les écoles, dans le sens d'une plus grande autogestion pédagogique. L'auteur de cet article interprète l'article 7, paragraphe 1 de la Loi fondamentale (« Le système scolaire dans son ensemble est placé sous la surveillance de l'État ») de la manière suivante : surveillance de l'État ne veut pas dire pour autant que l'école soit l'« affaire de l'État». Selon lui, ce terme impliquerait une large liberté d'organisation laissée aux écoles, accompagnée d'un «prudent contrôle » de la part de l'administration; on ne devrait avoir recours qu'en cas de nécessité extrême à des directives administratives. Cette conception du rôle de l'État concernant le système scolaire est nettement plus libérale que l'interprétation qu'en propose le Conseil d'État fédéral (Bundesverfassungsgericht) qui entend par « surveillance » : « ... la totalité des droits et des devoirs de l'État quand il s'agit d'organiser, de planifier, de diriger et de surveiller le bon fonctionnement du système scolaire ", mais aussi « le fait de décider du contenu des formations, des objectifs pédagogiques et des programmes» (Art. 34, 165 du Conseil). La juridiction en vigueur est encore plus claire à ce sujet (Art. 30, 59, 377) :

«L'organisation de l'école, sa structure générale, l'établissement des structures des systèmes de formation, les contenus et les programmes des différents cursus, la détermination des objectifs ainsi que l'évaluation permettant de savoir si et dans quelle mesure ces objectifs ont été atteints par les élèves font intégralement partie du domaine de l'État qui doit les mettre en œuvre ».

Du point de vue du droit constitutionnel, les aspirations à une plus grande responsabilité des écoles se heurtent à des limites bien précises. Le terme, très répandu, d'autonomie est un choix malheureux, de l'avis général, car l'autonomie, en matière de droit, ne peut 
s'appliquer qu'à une personne juridique ; les écoles sont des "établissements publics qui n'ont pas la jouissance de droits civils». On a également fixé aux modèles d'« autogestion» des écoles des limites juridiques strictes, dans le respect de la Constitution $^{25}$.

Cet arrière-plan juridique permet de comprendre pourquoi les débats qui ont eu lieu à propos de l'autonomie de l'école dans la République fédérale, pendant des décennies, tournaient, le plus souvent, autour de la définition de nouvelles normes juridiques appropriées. Il est intéressant de constater que les efforts pour libéraliser l'école, d'un côté, et l'affirmation de l'autorité de l'État pour tout ce qui concerne son organisation, de l'autre côté, ne suivent pas dans les programmes des partis politiques le schéma habituel gauche/droite. Les syndicats d'enseignants allemands ne se sont livrés à aucune démonstration de force pour mettre fin au statut de fonctionnaire des enseignants, bien que les fonctionnaires soient de toute évidence limités dans leur liberté et, par là, dans leur autonomie (le terme étant ici pertinent). Le système scolaire privé, par ailleurs, qui accorde les plus grands espaces de liberté à l'autogestion des écoles, est plus largement représenté dans les Länder traditionnellement conservateurs, parce que ces derniers sont dirigés par des gouvernements marqués confessionnelle ment, pour plus des deux tiers, principalement catholiques ${ }^{26}$.

Ces dernières années, les discussions ont été à nouveau plus vives. Une des raisons, et non des moindres, était la révision des lois concernant l'école dans les deux Länder de la Hesse et de la Basse-Saxe (en 1992 et, dans une certaine mesure, en 1993), où l'on prévoyait d'octroyer une plus grande part de responsabilité aux écoles. Le fait que ce soit l'État qui donne l'impulsion vers une plus grande autonomie des écoles, aussi paradoxal que cela puisse paraître à première vue, est cependant compréhensible si l'on passe en revue les différents arguments en faveur de cette autonomie afin de pouvoir les comprendre dans leur contexte historique ${ }^{27}$. A la fin des années soixante, les débats autour de l'autonomie de l'école faisaient partie d'un mouvement politique général revendiquant la cogestion dans tous les domaines de la vie. Il y avait à la base l'idée d'émancipation avec comme objectif la " démocratisation de la société ». Cette idée a pris place, même si c'était sous une forme affaiblie et en l'adaptant au domaine spécifique de la pédagogie, parmi les objectifs éducatifs de l'école. Mais le but de l'éducation - c'est-à-dire de former un individu, majeur et responsable, qui agisse en ayant clairement conscience des choses, tel qu'on le retrouve dans les plans-cadres et les programmes de tous les Länder-présuppose logiquement qu'à l'école aussi on s'exerce à appliquer les règles démocratiques qui permettent de régler les conflits. C'est de là que partent toutes les formes de participation des parents et des élèves, leur cogestion, dont les organes, certes, en raison de leurs compétences très limitées, n'ont pu jouer qu'un rôle très inférieur à ce qu'on avait pu entendre à l'origine par la pratique de la cogestion, pratique qui servait de modèle à leur participation.

26 La réactivation des débats concernant l'autonomie de l'école dans le milieu des années quatre-vingt, dont on peut penser qu'elle a été, pour une part essentielle, à l'initiative d'un certain nombre de lois au niveau des Länder, ne remonte pas à l'époque de la renaissance de ces idées d'émancipation, mais vise de façon toute prosaïque, comparativement, à une plus grande efficacité dans l'organisation de l'école (Commission de l'éducation, Rhénanie-du-Nord- Westphalie). Il ne faut pas prendre ce terme d'efficacité uniquement au sens économique, mais aussi pédagogique et organisationnel ${ }^{28}$. C'est pourtant cet aspect économique qui a le plus attiré l'attention. En se plaçant dans la 
perspective de l'« école-entreprise" on est obligé, premièrement, d'apporter une réponse à la question de savoir quel "produit » l'école peut bien offrir; deuxièmement, grâce à l'analogie entre la façon de gérer l'école et le management d'une entreprise, il devient transparent qu'à côté d'une logique de gestion aux mains d'une administration hiérarchisée, il pourrait y avoir aussi d'autres alternatives concernant la manière de diriger les écoles, capables de venir à bout des obstacles que la bureaucratie dresse sur le chemin qui mène à la flexibilité des directions d'école. On ne doit pas perdre de vue, aussi convaincante que puisse paraître cette argumentation au premier abord en raison de sa simplicité - allant dans le sens d'un préjugé largement répandu sur la mentalité des directeurs et plus particulièrement des professeurs - que ce n'est pas un hasard si cette façon de voir les choses a le vent en poupe à une époque comme celle-ci, où les budgets publics sont soumis à une politique d'austérité particulièrement contraignante, unique dans l'histoire de la République fédérale. Le soupçon que l'État se serve de l'autonomie de l'école comme d'un instrument lui permettant d'économiser de l'argent en adoptant une démarche soi-disant innovante et rende les écoles, devenues autonomes, responsables de mesures drastiques d'amputation du budget de l'éducation, n'est pas loin.

On ne peut nier, malgré tout, que le fait de se placer du point de vue de la gestion des entreprises a mis un nouvel accent sur les débats au sujet de l'autonomie, un accent capital ; on a laissé de côté le problème de la structure de l'école pour voir comment il était possible concrètement de mettre cette autonomie en œuvre dans les écoles. De la sorte, les limites pratiques d'une autogestion de l'école, même si elle englobait aussi le domaine financier, étaient clairement tracées: un directeur d'école n'est pas un commerçant. L'organisation d'une école ne fait pas appel aux mêmes structures qu'une entreprise, où le travail s'effectuerait dans différents secteurs (achat, comptabilité, etc..) dont le fonctionnement et le développement entraîneraient des coûts considérables. Pour une (auto-) gestion de l'école efficace ce serait par contre une nécessité absolue. L'école est en effet déchargée, de façon significative, de telles tâches grâce à la structure administrative si souvent décriée.

Toujours en se plaçant du point de vue de la gestion des entreprises, un autre point se dégage, lorsqu'on compare les structures de communication d'une entreprise avec le réseau, en règle générale peu développé, des échanges entre les enseignants. Rolff ${ }^{29}$ souligne que les enseignants jouissent dans leur pratique pédagogique d'une «autonomie individuelle» :

«Ils sont libres de tout contrôle de la part de leurs collègues; les directeurs d'école n'assistent à leurs cours que pour établir un rapport sur leur façon d'accomplir leur service, ce qui se produit rarement. L'inspection contrôle encore plus rarement leur enseignement, en fait essentiellement à l'occasion d'une promotion ».

Par contre, le modèle que lui-même propose, pour une meilleure organisation de l'école, d'une autonomie corporative dont l'objectif serait le développement de structures organisationnelles prévoit « comme élément moteur de ce développement des équipes (d'enseignants) coopérant et formant entre elles un réseau». Cette autonomie corporative tend à poser des limites à l'autonomie individuelle de l'enseignant - et tout particulièrement en ce qui concerne l'évaluation, lorsqu'on doit faire intervenir dans un projet de cette nature des éléments évaluatifs s'inspirant de la gestion moderne des entreprises. Quand une école veut emprunter de nouvelles voies pédagogiques, il est indispensable de définir des critères d'évaluation, au moins pour le fonctionnement interne, qui renseigneront sur le succès ou l'échec du changement de structure. Il est 
nécessaire, ici aussi, de s'entendre, au niveau interne, sur ce que doit être l'output de l'école et de son enseignement.

Il est indéniable que les discussions au sujet de l'autonomie de l'école dans la République fédérale ont suivi ces dernières années une évolution caractérisée par le fait qu'on a cessé de mettre l'accent sur des problèmes qui relèvent du niveau macro-juridique, les questions, par exemple, de droit constitutionnel et de droit administratif. On s'est demandé au contraire comment, concrètement, on pourrait utiliser de façon plus productive, dans les limites juridiques connues, la liberté d'action et d'organisation pédagogique déjà existante dans les écoles. Les arguments utilisés dans le débat sur l'autonomie ont exercé leur pression dans une autre direction; cela permet de comprendre pourquoi les gouvernements des Länder sont davantage prêts à faire des concessions, indépendamment de leur couleur politique, pourquoi ils ont une attitude plus positive qu'il y a encore deux décennies envers les initiatives sérieuses, mûrement réfléchies, qui peuvent être prises du côté des écoles. Pour rendre compte de cette évolution, Vogel a proposé (en 1995) le concept d' « entente au niveau interne » de l'école (Selbstverständigung) qui - à la différence des autres termes comme «autonomie » et "autogestion" - offrirait une plus grande liberté d'action du point de vue de la Constitution, dans la limite où la législation le permettrait au niveau des Länder. Que les écoles s'acheminent ainsi sur un mode plutôt pragmatique, pas à pas, vers une complète prise de responsabilités dans les années à venir, correspond bien à la structure fédérale du système scolaire au niveau de la république.

\section{L'extension des contrôles de la réussite ${ }^{30}$}

\section{Les directives}

Le système scolaire de la République fédérale, sans oublier l'enseignement supérieur, est organisé de manière politico-administrative. Des moyens en personnel et en matériel sont fournis, des programmes de régulation sont envoyés- on entend par là l'ensemble des instructions juridiques et administratives déterminant les objectifs et les méthodes dans l'éducation publique. Au cœur de ces instructions officielles (Regulative Programme) figurent les dispositions légales qui fixent les structures organisationnelles du système scolaire et les directives des diverses administrations scolaires: les programmes d'enseignement, les horaires, les directives concernant l'homologation des manuels scolaires et celles d'après lesquelles sont organisés les examens. Ils indiquent quels sont les objectifs à atteindre tout en laissant une importante marge de manœuvre quant au choix des chemins qui mèneront à ces objectifs. Comment savoir si et comment ces objectifs sont atteints? La question demeure, le plus souvent, ouverte. Les instructions dont dépendent l'organisation des examens et l'évaluation ne présentent de critères transparents pour l'organisation du contrôle des résultats scolaires que dans quelques domaines sensibles. Dans le système éducatif de la République fédérale, les objectifs pédagogiques qui ont été fixés servent aussi, le plus souvent, de référence pour les résultats effectivement obtenus. Ce sont les objectifs fixés de façon formelle - et non pas les connaissances et les compétences réelles - qui font l'objet de discussions publiques, ils sont les instruments de mesure de l'action ministérielle. Cette manière de concevoir l'organisation politico-administrative $\mathrm{du}$ système éducatif s'accorde mal avec l'organisation de contrôles systématiques de la réussite scolaire, étant donné que cela fait 
aussi toujours courir le risque d'une dévaluation des objectifs fixés. Il n'est en aucun cas assuré, par exemple, qu'un programme d'enseignement plus exigeant dans une discipline entraine aussi, effectivement, un plus haut niveau d'exigence jusque dans la transmission du savoir en cours. Avec un tel arrière-plan, il n'est pas étonnant que des examens centralisés (comme c'est le cas en France) ou même les large scale assessments programs (aux États-Unis) restent des exceptions.

Renoncer aux contrôles centralisés de la réussite scolaire ne signifie pas pour autant, bien évidemment, qu'il n'y ait plus aucun instrument de contrôle qualitatif dans le système éducatif. Au niveau des Länder, les programmes d'enseignement, les horaires, les instructions concernant l'organisation des examens déterminent le niveau des objectifs à atteindre dans les différentes formations. Le contrôle des résultats scolaires sert avant tout d'indicateur, en retour, pour les élèves, sur ce qui a été étudié en cours. On souhaite établir ainsi des comparaisons à l'intérieur d'un groupe d'élèves, sans chercher à comparer des groupes entre eux, à plus grande échelle. Les instructions concernant l'organisation des examens ne prennent un caractère plus obligatoire que si elles sont votées au niveau fédéral par la Conférence des ministres de l'Éducation. À deux carrefours particuliers du système éducatif, à la fin de la scolarité obligatoire et à la fin du second cycle du secondaire, les ministres de l'Éducation ont défini, dans chaque discipline, des standards et des exigences concernant les examens communs aux différents Länder (les standards pour le premier cycle du secondaire et les exigences, unitaires, concernant l'Abitur). Il n'y a, par contre, aucun règlement unitaire, passant les frontières des Länder, pour ce qui est des contrôles généraux qualitatifs dans la République fédérale. Quelques Länder prescrivent des examens centralisés en fin de cycle. L'exemple le plus connu est celui de l'Abitur centralisé, organisé dans le BadeWurtemberg, en Bavière et dans la Sarre. D'autres Länder renoncent aux examens centralisés de fin de cycle car on est obligé de se limiter à un domaine essentiel de la matière étudiée, par souci d'honnêteté, à cause des matières à option obligatoire et des formations comportant une matière dominante. Ces Länder préfèrent demander aux établissements de définir des tâches mieux adaptées à ce qui a été effectivement enseigné, tâches qu'il faut, par contre, soumettre obligatoirement à l'accord de l'administration afin d'être certain qu'elles se situent bien au niveau exigé. Il est prévu, en même temps, une double correction des copies d'examen qui sont échangées entre les établissements. La question de savoir quelle méthode est la plus efficace pour s'assurer de la qualité d'une formation est un sujet de conflit permanent entre les Länder, les partis politiques et les groupements d'intérêts. Les examens standardisés qui permettraient d'observer l'évolution des choses à long terme restent inconnus dans le système scolaire allemand. C'est dans l'enseignement supérieur qu'on trouve le plus faible système de contrôle qualitatif. Une certaine standardisation, impliquant une coopération entre plusieurs universités, existe uniquement dans les disciplines où l'État organise des examens nationaux de recrutement. Le fait que, dans le système éducatif de la République fédérale, les règlements concernant les examens ne soient dans l'ensemble guère comparables entre eux, a pour conséquence des écarts extrêmes dans les différents domaines de la formation, de l'évaluation et du contrôle de la réussite. 


\section{La rupture avec la tradition}

33 On voit se dessiner ces derniers temps une rupture avec la tradition dans la philosophie de l'organisation politico-administrative du système éducatif de la République fédérale. Il $\mathrm{y}$ a deux points très différents à l'origine de cette évolution. L'attention s'est focalisée ces dernières années sur l'école, l'établissement scolaire pris isolément et sa structure pédagogique en tant que facteur de qualité, du point de vue éducatif. Il était question de mettre au point un standard, au niveau de l'établissement scolaire, et de s'assurer de sa valeur. Un tel programme impose des tâches supplémentaires au corps enseignant et lui confère de plus grandes responsabilités. Accorder de plus larges responsabilités à l'école parler d'autonomie ne serait pas pertinent étant donné la surveillance exercée ici par l'État - revient à faire gérer par l'école, à une époque où on dispose de moyens limités, une relative austérité, l'administration centrale dégageant ainsi en partie ses responsabilités et échappant davantage à la critique ( $c f$. «Autonomie des écoles»). On peut en même temps attendre, en accordant ainsi un plus grand pouvoir de décision au niveau de chaque établissement scolaire, une meilleure adéquation de ce que l'école peut offrir aux conditions locales particulières. En réponse à la menace que pourrait alors constituer le particularisme dans le système éducatif, avec toutes les conséquences néfastes qu'entraînerait une diversification croissante des standards, il est question de procéder périodiquement, au niveau national, à des contrôles de vérification, qui devraient apporter la garantie que les standards de référence n'ont pas varié. Deux évolutions, en apparence contraires, s'affrontent et en même temps, pour ainsi dire, s'apportent mutuellement leur soutien.

Cette tendance à organiser des contrôles qualitatifs de réussite qui dépassent les frontières des Länder correspond à la nécessité de parvenir à une intégration du système éducatif européen. En outre, on se trouve contraint et forcé de le faire sous la pression croissante de la concurrence internationale, à laquelle les systèmes éducatifs nationaux sont de plus en plus soumis. L'OCDE, en particulier, dont les programmes concernant les indicateurs accordent une importance particulière aux indicateurs de performance, est là un élément moteur puissant. Dans cette constellation, où l'on voit les efforts de décentralisation se conjuguer avec un accroissement de la concurrence internationale, la "Troisième enquête internationale sur les mathématiques et les sciences" (TIMSS) de l'TEA (International Association for the Evaluation of Educational Achievement) - dont les résultats en ce qui concerne l'Allemagne sont peu flatteurs - a attisé à nouveau, d'une façon surprenante, la discussion sur les contrôles nationaux de la réussite scolaire. Pour la première fois depuis trente ans, la TIMSS a mis à la disposition du public des informations sur la concurrence internationale concernant la République fédérale. Elles montrent à quel point le groupe d'élèves qui obtient de très faibles résultats est important et, d'autre part, attirent l'attention sur le problème de l'équité distributive tenant au fait que des différences considérables existent entre les différents Länder sur le plan des résultats scolaires. Ce qui a eu pour conséquence de faire apparaître une constellation politique dans laquelle la Conférence des ministres de l'Éducation a récemment rompu avec la façon antérieure de diriger le système éducatif concernant la régulation de l'input: la République fédérale va prendre part aux enquêtes périodiques sur les résultats scolaires de l'OCDE et mettre en place, dans les années qui viennent, un National Assessment Program. 


\section{NOTES}

2. Rédigé par Dirk Konietzka.

3. Geißler, K.A. (1991). «Das duale System der industriellen Berufsausbildung hat keine Zukunft ». Leviathan, 19, 68-77.

Greinert, W.-D. (1994). « Berufsausbildung und sozio-ökonomischer Wandel. Ursachen der "Krise des dualen Systems" der Berufsausbildung ». Zeitschrift fur Pädagogik, 40, 357-372.

Lempert, W. (1995). « Das Märchen vom Niedergang des "dualen Systems" ». Zeitschrift fur Berufsund Wirtschaftspädagogik, 91, 225-231.

Liesering, S., Schober K. \& Tessaring M. (Hg.) (1994). Die Zukunft der dualen Berufsausbildung. Eine Fachtagung der Bundesanstalt fur Arbeit (BeitrAB 186).

Mayer, K.U. (1995). «Das duale System der beruflichen Ausbildung - Ultrastabilität oder Auflösung?». Hoff, E.-H./ Lappe, L. (Hg.), Verantwortung im Arbeirsleben, 89-113.

4. Kutscha, G. (1992). "Das duale System der Berufsausbildung in der Bundesrepublik Deutschland - ein auslau-fendes Modell ?». Die berufsbildende Schule, 44, 145-156.

5. BIBB (Bundesinstitut für Berufsbildung) (Hg.) (1995) : Die anerkannten Ausbildungsberufe 1994, Bielefeld. Münch, J. (1994). Das Berufsbildungssystem in der Bundesrepublik Deutschland. Berlin: CEDEFOP.

6. Sengenberger, W. (1987). Struktur und Funktionsweise von Arbeitsmärkten. Die Bundesrepublik Deutschland im internationalen Vergleich. Frankfurt/M. Campus.

Blossfeld, H.-P. \& Mayer, K.-U. (1988). «Arbeitsmarktsegmentation in der Bundesrepublik Deutschland. Eine empi-rische Überprüfung von Segmentationstheorien aus der Perspektive des Lebenslaufs ». Kölner Zeitschrift fur Soziologie und Sozialpsychologie, 40, 262-283.

Konietzka, D. (1997). "Verberuflichung" und "Destandardisierung". Stabilität und Wandel der Ausbildungserfahrungen und Berufsintegration der Kohorten 1919-21 und 1959-61 in der Bundesrepublik Deustchland. " Dissertation. Freie Universitat Berlin.

7. Op. cit., note 3 .

8. Tessaring, M. (1993). «Das duale System der Berufsausbildung in Deutschland: Attraktivität und Beschäftigungspersperktiven». Mitteilungen aus der Arbeitsmarkt- und Berufsforschung. 26, 131-161.

Arnold, R. (1994). «Die Zukunft der dualen Berufsausbildung». Kuratorium der deutschen Wirtschaft fur Berufsbildung (Hg.). Kaufmännische Ausbildung im Wettbewerb, Bonn.

9. Berufsbildungsbericht 1992. Bundesministerium für Bildung und Wissenschaft (Hg.) (1992), Bad Honnef.

10. Kutscha, G. (1994). «Modernisierung der Berufsbildung im Spannungsfeld von Systemdifferenzierung und Koordination». In Buttler, F., Czycholl, R. \& Pütz, H. (Hg.). Modernisierung beruflicher Bildung vor den Ansprüchen von Vereinheitlichung und Differenzierung (BeitrAB 177), 40-56.

11. Lutz, B. (1991). « Herausforderungen an eine zukunftsorientierte Berufsbildungspolitik ». In : BIBB (Bundesinstitut fur Berufsbildung) (Hg.): Die Rolle der beruflichen Bildung und Berufsbildungsforschung im interna-tionalen Vergleich, Tagungsdokumentation, 27-36.

12. Mayer, K.-U. (1996). «Ausbildungswege und Berufskarrieren» in : BIBB (Bundesinstitut fur Berufsbildung) (Hg.): Forschung im Dienst von Praxis und Politik, Dokumentation der Festveranstaltung zum 25-jährigen Bestehen des Bundesinstituts fur Berufsbildung, 113-145.

13. Op. cit., note 11 .

Revue internationale d'éducation de Sèvres, 15 | 2013 
14. Hamilton, S.F. \& Hurrelmann, K. (1993). « Auf der Suche nach dem besten Modell für den Übergang von der Schule in den Beruf - ein amerikanisch-deutscher Vergleich ». Zeitschrift für Sozialisationsforschung und Erziehungssoziologie, 13, 194-207.

Schauman, F. (1993). « Gleichwertigkeit von Berufs- und Allgemeinbildung - Zukunftschance der beruflichen Bildung». BWP, 22, 11-19.

15. Lutz, B. \& Voß, G. (1992). «Subjekt und Strukturwandel - Versuch der Neubestimmung einer soziologischen Schlüsselbeztehung am Beispiel des Facharbeiters » in : Heinz, W.R. und Lutz, B. (Hg.), Modernisierungsprozesse von Arbeit und Leben, Sonderforschrungsbereich 333 der Universität München. Mitteilungen Sonderheft 1, 77-89.

16. Adler, T., Dybowski, G. \& Schmidt, H. (1993). «Kann sich das duale System behaupten?». Argumente für eine zukunftsorientierte Berufsausbildung. BWP, 22, 3-10.

17. Rédigé par Olaf Köller.

18. Ce système se distingue, mutatis mutandis, par un cycle primaire indifférencié de quatre ans suivi d'un cycle de cinq à six ans, ce premier cycle du secondaire s'effectuant dans différentes écoles : Hauptschule, Gesamtschule, Realschule, Gymnasium (lycée).

Arbeitsgruppe Bildungsbericht am Max-Planck-Institut für Bildunsforschung (1994). Das Bildungswesen in der Bundesrepublik Deutschland. Strukturen und Entwicklungen im Überblick. Reinbek : Rowohlt.

19. Baumert, J., Roeder, P.M., Sang, F. \& Schmitz, B. (1986). « Leistungsentwicklung und Ausgleich von Leistungsunterschieden in Gymnasialklassen ». Zeitschrift für Pädagogik, 32, 639-660.

Jaegger, R.M. (1994). «Evaluating policy inferences drawn from international comparisons of students' achievement test performances ». Studies in Educational Evaluation, 20, 23-39.

20. Schnabel, K., Baumert, J. \& Roeder, P.M. (1996). «Zum Wandel des Schulsystems in den neuen Bundeslàndern ». Neue Sammlung, 36, 531-544.

21. Il convient de noter que ce soutien particulier apporté aux élèves obtenant des résultats médiocres, comme d'ailleurs les moyens utilisés pour encourager les meilleurs élèves, avait lieu en dehors de l'école.

22. Baumert, J., Lehmann, R. et a). (1997). TIMSS - Mathematisch- naturwissenschaftlicher Unterricht im natio-nalen Vergleich. Opladen : Leske + Budrich.

23. Rédigé par Kai Schnabel.

24. Becker, H. (1954/1993). "Die verwaltete Schule». Recht der Jugend und des Bildungswesens (Wiederabdruck), 41, 130-147.

25. Avenarius, H. (1995). "Verfassungsrechtliche Grenzen und Möglichkeiten schulischer Selbstverwaltung ». In : Daschner, P., Roff, H.-G. \& Stryck, T., Schulautonomie, Chancen und Grenzen. Weinheim : Juventa.

26. Op. cit., note 18.

27. Richter, I. (1995). « Theorien der Schulautonomie ». In : Daschner, P., Roff, H.-G. \& Stryck, T., Schulautonomie, Chancen und Grenzen. Weinheim : Juventa.

28. Op. cit., note 26 .

29. Rolff, H.-G. (1995). «Autonomie als Gestaltungs-Aufgabe ». In : Daschner, P., Rolff, H.-G. \& Stryck, T., Schulautonomie, Chancen und Grenzen. Weinheim : Juventa.

30. Rédigé par Jürgen Baumert. 


\section{RÉSUMÉS}

Malgré la liberté d'action reconnue aux différents Länder en matière d'éducation, certains thèmes, liés aux transformations politiques et socioculturelles en Allemagne, se manifestent de façon récurrente: la crise du système dual, l'autonomie des écoles, l'évaluation nationale des performances et, bien sûr, la réforme du système scolaire dans les nouveaux Länder.

\section{INDEX}

Index géographique : Allemagne

Mots-clés : autonomie, établissement d'enseignement, politique éducative, réforme de l'enseignement, système éducatif

\section{AUTEURS}

\section{JÜRGEN BAUMERT}

Professeur, directeur du Max-Planck Institut, Berlin, Allemagne

\section{OLAF KÖLLER}

Psychologue chercheur au Max-Planck Institut, Berlin, Allemagne

\section{DIRK KONIETZKA}

Psychologue chercheur à l'université Rostock, Allemagne

\section{KAI SCHNABEL}

Psychologue, chercheur au Max-Planck Institut, Berlin, Allemagne 